\title{
Outcomes From a Hybrid Implementation- effectiveness Study of the Living Well During Pregnancy Tele-coaching Program for Women at High Risk of Excessive Gestational Weight Gain
}

Susan de Jersey ( $\nabla$ susan.dejersey@health.qld.gov.au )

Royal Brisbane and Women's Hospital

Nina Meloncelli

The University of Queensland

Taylor Guthrie

Royal Brisbane and Women's Hospital

Hilary Powlesland

Royal Brisbane and Women's Hospital

Leonie Callaway

Royal Brisbane and Women's Hospital

Angela T Chang

Royal Brisbane and Women's Hospital

Shelley Wilkinson

The University of Queensland

Tracy Comans

The University of Queensland

Elizabeth Eakin

The University of Queensland

\section{Research Article}

Keywords: implementation study, pregnancy, gestational weight gain, nutrition, telephone counselling, dietitian

Posted Date: January 3rd, 2022

DOI: https://doi.org/10.21203/rs.3.rs-1143454/v1

License: (9) (1) This work is licensed under a Creative Commons Attribution 4.0 International License. Read Full License 


\section{Abstract}

\section{Background}

Excess gestational weight gain (GWG) is associated with short-term perinatal complications and longer term cardiometabolic risks for mothers and their babies. Dietitian counselling and weight gain monitoring for women at risk of high pregnancy weight gain is recommended by clinical practice guidelines. However, face-to-face appointments, during a time with high appointment burden, can introduce barriers to engaging with care. Telephone counselling may offer a solution. The Living Well during Pregnancy (LWdP) program is a dietitian-delivered telephone coaching program implemented within routine antenatal care for women at risk of excess GWG. This program evaluation used a hybrid implementationeffectiveness design guided by the RE-AIM framework reports on the primary outcomes (reach, adoption, implementation, maintenance) and secondary outcomes (effectiveness) of the LWdP intervention.

\section{Methods}

The LWdP program evaluation compared data from women participating in the LWdP program with a historical comparison group (pregnant women receiving dietetic counselling for GWG in the 12 months prior to the study). The primary outcomes were described for the LWdP program. Between group comparisons were used to determine effectiveness of achieving appropriate GWG and pre and post intervention comparisons of LWdP participants was used to determine changes to dietary intake and physical activity.

\section{Results}

The LWdP intervention group ( $n=142)$ were compared with women in the historical comparison group $(n=49)$. Women in the LWdP intervention group attended $3.4(95 \% \mathrm{Cl} 2.9-3.8)$ appointments compared with $1.9(95 \% \mathrm{Cl}, 1.6-2.2)$. GWG was similar between the two groups, including the proportion of women gaining weight above the Institute of Medicine recommendations ( $70 \%$ vs $73 \%, p=0.69$ ). Within group comparison showed that total diet quality, intake of fruit and vegetables and weekly physical activity were all significantly improved from baseline to follow-up for the women in the LWdP, while consumption of discretionary food and time spent being sedentary decreased (all $p<0.05$ ).

\section{Conclusion}

The LWdP program resulted in more women accessing care and positive improvements in diet quality, intuitive eating behaviours and physical activity. It was as effective as face-to-face appointments for GWG, though more research is required to identify how to engage women earlier in pregnancy and reduce appointment burden.

\section{Background}


Excess gestational weight gain (GWG) is one of the most common adverse outcomes of pregnancy, experienced by half to three quarters of women in developed countries [1, 2]. It is associated with hypertensive disorders of pregnancy, gestational diabetes mellitus (GDM), instrumental and caesarean deliveries, large for gestational age babies and macrosomia [1, 3-5]. Longer term consequences of gaining more weight than recommended during pregnancy include future childhood overweight for offspring and post-partum weight retention leading to obesity in mothers [6, 7]. Each $1 \mathrm{~kg}$ of excess GWG has been associated with and $3 \%$ increase in childhood obesity [8]. Women with excess GWG retain on average 2 to $4 \mathrm{~kg}$ per pregnancy, further contributing to high body weight in women of a reproductive age $[6,7]$.

Evidence is now well established that theory-based behaviour change nutrition interventions coupled with weight monitoring are effective in reducing GWG $[9,10]$ and can impact on selected pregnancy outcomes such as GDM and hypertension [11, 12]. In line with this many clinical practice guidelines recommend consideration of[13], or a referral to a dietitian [14] to support women, particularly those with an increased pre-pregnancy body mass index (ppBMI), achieve a healthy pregnancy weight gain. However, uptake to such services in routine care has been poor, particularly with face-to-face care [15-18].

Telephone counselling has the potential to provide individualised intensive support to those women at high risk of excess GWG without the need for additional attendance at hospital or antenatal clinics and has been an effective and cost-effective solution for weight management outcomes in many adult populations [19-21]. While telephone based interventions have been trialled in pregnancy [22], there has been limited evaluation of the implementation or effectiveness associated with translation into practice [23].

The Living Well during Pregnancy (LWdP) program was a dietitian delivered telephone coaching program implemented within routine antenatal care. A core program component was motivational interviewing with a foundation in behaviour change principles.

Using the RE-AIM framework [24] to guide evaluation, here we report on the primary outcomes (program reach, adoption, implementation, maintenance) and secondary (effectiveness) outcomes including behavioural and anthropometric changes.

\section{Methods}

\section{Setting}

The study was conducted at a large, tertiary metropolitan hospital that delivers approximately 4,500 babies per year. Detailed methods for the LWdP program and evaluation have been previously reported [25].

\section{Study design}


The LWdP program was evaluated using a hybrid implementation-effectiveness design [26]. Data from consenting participants enrolled in the LWdP program were compared with a historical comparison group of pregnant women referred for dietetic support for healthy pregnancy weight management.

\section{Participants and referral pathways}

Participants were clinician or self-referred to the LWdP program for dietetic care between February 2018 and March 2019.

\section{Eligibility criteria}

Included women were those aged $\geq 16$ years, without pre-exiting diabetes and referred for dietetic weight management care during the study period. Specifically, women with a pre-ppBMI greater than $25 \mathrm{~kg} / \mathrm{m}^{2}$, or a ppBMI less than $25 \mathrm{~kg} / \mathrm{m}^{2}$ and had been gaining weight above the Institute of Medicine (IOM) [5] recommendations were accepted upon referral at any point in their pregnancy. Only participants who were able to speak and read English sufficiently to allow program participation were included. Those women who did not meet these criteria were provided with appropriate face-to-face dietetic care and did not participate in this evaluation. Women were referred to the program by their antenatal care provider or were able to self-refer into the program through a dedicated program website.

The historical comparison group were pregnant women who met the inclusion criteria, referred for dietetic care in the 12 months prior to the study period.

\section{The Living Well during Pregnancy program}

A detailed description of the LWdP program is found elsewhere [25]. Briefly, the program was aimed at supporting women at high risk of excess weight gain during pregnancy to achieve GWG within recommendations [5]. The program was grounded in Social Cognitive Theory constructs of self-efficacy, social support and outcome expectancies [27] and emphasised developing skills in behaviour change strategies - goal setting, self-monitoring, identification of potential barriers and problem solving, identifying social support, stimulus control, mindful eating, positive self-talk and self-reward. The goal was for women to track within their healthy GWG for their ppBMI based on IOM recommendations [5] through changing eating and activity behaviours. This included healthy eating, and physical activity, consistent with dietary and physical activity guidelines for pregnancy. Women were eligible for up to 10 telephone coaching calls over their pregnancy and were provided with a participant workbook. Accredited Practising Dietitians with experience in providing antenatal care services who had undergone additional training in motivational interviewing were trained specifically for the delivery of the program. A key component of the program was continuity of care through allocation of the same Dietitian throughout the duration of the program. The program was adapted for pregnancy from the Healthy Living after Cancer program [28].

\section{Historical comparison group}


The historical comparison group were provided with usual dietetic care. Women were offered an individual appointment with the dietitian in a dedicated clinic within the maternity outpatient department. An initial appointment was booked for 40 minutes with review appointments allocated 20 minutes. There was no guarantee of the same dietitian at a review appointment, and these were booked based on clinical judgement in negotiation with the woman. Clinical practice guidelines at the time recommended women had a consultation with a dietitian to support healthy eating in line with the Australian Dietary Guidelines and encourage adherence to weight gain recommendations [14]

\section{Data collection}

Data collection is reported in greater detail elsewhere [25]. The data for the historical comparison group were extracted from administrative (referral and attendance), medical and pregnancy handheld records (anthropometric and demographic). Data for the LWdP intervention group was collected by study-trained dietitian research assistants using the Research Data Capture (REDCap) online data capture tool to record details of each telephone counselling session including progress, clinical information, and call

fidelity (self-reported after each call by clinician via checklist). Behavioural and anthropometric data were collected via an online questionnaire through a health service consumer portal, with the follow-up questionnaire containing additional participant satisfaction and feedback questions. Women who withdrew from the program were sent an online survey to assess opportunities for program improvement and reasons for withdrawal. Administrative data (both routinely collected and purposefully requested for the purposes of the study) were recorded in an excel spreadsheet. An online and paper-based staff survey was sent to all antenatal staff between December 2018 and January 2019. This survey examined staff satisfaction with the LWdP program and referral processes in addition to understanding of eligibility criteria and model of care.

The Fat and Fibre Behaviour Questionnaire (FFBQ) [29], a validated 20 item questionnaire to assess dietary behaviours was used to calculate Fat Index, Fibre Index and Total Diet Index (scored 1 - 5, with 5 indicating more optimal eating behaviours). The FFBQ was also used to describe the serves of fruits, vegetables, low fat dairy foods and discretionary foods consumed daily. The Intuitive Eating Scale-2 was used to measure participants ability to follow their physical hunger and satiety cues when determining when, what, and how much to eat [30]. The Active Australia Survey[31] was used to assess the frequency and duration of physical activity (walking, moderate and vigorous) in the past week. Total number of sessions and minutes of physical activity were treated as continuous variables. Sedentary behaviour (sitting time per week on weekdays and weekends), questions from the International Physical Activity Questionnaire was used to assess total sedentary time per week [32]. Behavioural outcomes were not available for the historical comparison group as they were not collected as part of routine care.

\section{Outcomes}

Primary outcomes were mapped to the RE-AIM framework measures of Reach and Representativeness, Adoption. Implementation, and Maintenance. The RE-AIM measure of Effectiveness was a secondary 
outcome which included anthropometric measures and behaviour change outcomes. Results have been reported in this order.

\section{Statistical analysis}

Analyses were performed using Statistical Package for Social Sciences (Version 20, SPSS Inc., Chicago, USA). All available data were included in analysis. Primary outcomes were reported descriptively. The adoption and reach primary outcomes were compared to the historical comparison group while the implementation outcomes were reported for the LWdP intervention group only. For the effectiveness outcome of GWG, the historical comparison group and LWdP intervention group were compared. GWG differences between the groups was analysed according to pre-pregnancy body mass index (continuous

and according to meeting the IOM recommendations). Within group GWG differences (LWdP group only) were analysed according to: per protocol and intention-to-treat (where per protocol was defined by the research team as completion of 4 or more telephone counselling sessions) and whether counselling commenced early in the pregnancy ( $\leq 16$ weeks gestation) vs after 16 weeks gestation. Logistic regression was used to calculate the odds ratio of women in the LWdP intervention group achieving the IOM GWG recommendations and adjusting for pre-specified cofounders. Missing GWG data for the LWdP group occurred for less than $15 \%$ of participants $(n=20)$. Therefore, mean substitution or data imputation was not performed.

For all other effectiveness outcomes (diet and physical activity behaviours), baseline and follow-up questionnaire data were compared within the LWdP intervention group only and mean change or \% change were compared using paired-sample t tests. Comparisons between women completing both baseline and follow-up questionnaires and those who only completed the baseline questionnaire was analysed to understand whether there were differences in characteristics between completers and noncompleters. Due to the nature of the data collected in the behavioural questionnaire, data could not be imputed. Only data for those women who completed both questionnaires were include for analysis.

Mean and standard deviation (SD) were reported for normally distributed data while median and interquartile range (IQR) for skewed data. Differences between groups were assessed using independent sample t-tests for continuous variables and chi-squared for categorical variables.

\section{Results}

\section{Reach and representativeness}

Referral, participation and exclusion rates for both groups are shown in Figure 1. During the study period, 2532 women receiving antenatal care at the study hospital had a ppBMl $>25 \mathrm{~kg} / \mathrm{m}^{2}$, with an estimated $13 \%$ of women eligible to receive the LWdP program referred, compared to $3 \%$ of women referred in the historical comparison group $(\mathrm{n}=2331)$. Women commenced dietitian appointments at similar weeks gestation across both groups. Participant 'characteristics of the historical comparison and LWdP 
intervention groups are shown in Table 1. No meaningful (>10\%) between group baseline differences were apparent.

Table 1

Maternal characteristics of participants in the LWdP intervention and historical comparison group

\begin{tabular}{|c|c|c|c|}
\hline & $\begin{array}{l}\text { LWdP intervention group } \\
(n=142)\end{array}$ & $\begin{array}{l}\text { Historical comparison } \\
\text { group }(n=49)\end{array}$ & $P$ \\
\hline Age, years & $31.6(5.2)$ & $29.8(6.3)$ & .05 \\
\hline Country of birth (Australia) & $88(62 \%)$ & $27(55 \%)$ & .40 \\
\hline Nulliparous & $81(57 \%)$ & $34(69 \%)$ & .13 \\
\hline \multicolumn{4}{|l|}{ Education } \\
\hline Primary/ high school & $46(32 \%)$ & - & \\
\hline Trade certificate or diploma & $32(23 \%)$ & - & \\
\hline $\begin{array}{l}\text { University degree (including } \\
\text { postgraduate) }\end{array}$ & $64(45 \%)$ & - & \\
\hline Pre-pregnancy BMI kg/m² & $30.6(6.6)$ & $33.4(7.9)$ & .02 \\
\hline Underweight (BMI<18.5 kg/m²) & $1(0.7)$ & 0 & \\
\hline $\begin{array}{l}\text { Normal weight (BMI18.5-24.9 } \\
\mathrm{kg} / \mathrm{m}^{2} \text { ) }\end{array}$ & $24(17 \%)$ & $4(8 \%)$ & \\
\hline Overweight (BMI25-29.9) kg/m²) & $49(35 \%)$ & $14(29 \%)$ & \\
\hline Obese $\left(\mathrm{BMI}>30 \mathrm{~kg} / \mathrm{m}^{2}\right)$ & $68(48 \%)$ & $31(63 \%)$ & .23 \\
\hline $\begin{array}{l}\text { Gestation at first dietitian } \\
\text { appointment, weeks }\end{array}$ & $21.5(6.2)$ & $22.9(5.0)$ & .18 \\
\hline \multicolumn{4}{|c|}{ LWdP Living Well during Pregnancy; BMI Body Mass Index } \\
\hline \multicolumn{4}{|c|}{ Results shown as either $n(\%)$ (categorical) or mean (SD) (continuous) } \\
\hline
\end{tabular}

\section{Adoption}

Seventy-three percent $(n=270)$ of the women referred to the LWdP program were referred by midwives, $23 \%(n=86)$ were self-referrers, $1 \%(n=3)$ were referred by General Practitioners, $1 \%(n=3)$ by an Obstetrician $<1 \%(n=2)$ were by a dietitian. 


\section{Staff survey (referral practices and staff attitudes towards the program)}

Fifty-three staff answered the LWdP evaluation survey. Forty-three (77\%) respondents were health professionals (42 were midwives/nurses, 2 were Obstetrics and Gynecology doctors) and the remaining $19 \%(n=10)$ of respondents were administration officers.

Sixty percent $(n=6)$ of the of the administration officers and $86 \%(n=37)$ of health professional respondents agreed that they understood what the LWdP program was and $100 \%$ correctly identified the LWdP description. Education sessions or emails from dietitians, posters/flyers and dietitian referral forms were the most common ways the administration officers learnt about the program and three health professionals said that they learnt about the program from their patients. Fifty percent $(n=5)$ of administration officers agreed that the referral processes were easy. Almost all health professionals agreed that they were aware of who was eligible, how to refer and that referral to the program was easy. More than half of the health professionals (67\%) reported they 'always' or 'often' referred eligible women. Two respondents did not feel comfortable recommending the program. Three administration officers thought they had enough knowledge of the program to answer women's questions while a further three did not feel they had enough knowledge.

Eighty-three percent $(n=44)$ of survey respondents did not feel as though the burden of appointments or appointment timing would be a barrier to referring to the program. However, more than half $(n=24)$ felt that a barrier to referral (always, often or sometimes as responses) was that women don't see weight as a priority. Seventeen respondents thought that women sometimes (or often) had a negative view of dietitians.

\section{Implementation and maintenance}

LWdP delivery required an $80 \%$ full time equivalent dietitian coach (senior level). A second coach covered vacation time if leave extended beyond 2 weeks. The coaching calls were offered across four days per week with two early morning starts (7.30am-3.30pm) and two later evening (9:30am -6:00pm) to enable maximum participation. The time allocation and LWdP program continues to be offered within routine care to all women meeting the referral criteria at the study site. Of note from the staff survey, almost half $(n=18)$ of the respondents did not feel they were informed of the progress of women they referred to the program and the majority would be happy to receive an email or read patient progress notes to keep informed.

\section{Completion rates and number of appointments attended}

In the historical comparison group, the mean number of appointments was 1.9 (95\% $\mathrm{Cl}, 1.6-2.2)$. The greatest number of appointments attended by a single participant was five. The average number of LWdP 
appointments attended was $3.4(95 \% \mathrm{Cl}, 2.9$ - 3.8). One participant attended 11 appointments in total. The number of women attending at least four appointments (considered 'per protocol') was $58(41 \%)$. Eighty-four women (60\%) withdrew from the program before completing at least four appointments. Forty-seven women (34\%) attended one or fewer appointments. Women commencing pregnancy overweight had a tendency towards completing four or more appointments compared with women who commenced pregnancy at a normal or obese BMI ( $47 \%$ vs $36 \%$ and $38 \%$, respectively, $P=.55$, results not shown).

\section{Reasons for withdrawal}

Nine women completed the drop-out survey, with the most common reason $(n=4)$ for program withdrawal being 'I don't have enough time to complete calls'. Competing work priorities, early labour and wanting specific snack/meal suggestions were reasons for not completing the program.

\section{Implementation strategies}

Twenty-six implementation strategies were used to support service change ranging from accessing new funding to mandating change. A full description of each strategy is outlined in Additional file 1. Six months after the program commenced, a text message to each woman registering to birth at the hospital was sent with information about the program and a link to the self-referral website. In the three months before the text message was initiated there was an average of 47 visits to the website per month, increasing to 148 visits per month after the text message $(P=.03)$ with self-referrals increasing from 3 per month to 21 per month $(P<0.01)$.

\section{Coaching call fidelity}

Self-reported coaching call fidelity showed that all topics were covered as planned in the first coaching call for $95 \%$ of the participants, however there was no fidelity check undertaken for $15 \%(n=21)$ of participants. For each call, goal attainment, goal setting and pre-reading requirements were discussed with more than $80 \%$ of participants. Adherence to other planned topics of discussion declined with each subsequent call. For example, the topics discussed with more than $75 \%$ of participants in call two were dietary trackers, healthy diet and goal setting whereas reducing fat intake, food safety and healthy portions was discussed less than $50 \%$ of the time. For call three, the topics relating to physical activity were discussed with around half of the participants (or fewer) and women appeared to continue the discussions from call two rather than stick to the planned topics. Participants were keen to discuss certain topics earlier than were originally planned in the program's workbook or felt that certain sections didn't apply to them (for example, physical activity). By call four, fidelity checks were performed on less than $60 \%$ of calls ( $n=36$ out of 60 participants) and only $19 \%(n=7)$ of participants discussed planned topics (healthy GWG, meal planning, eating at the right times and tracking weight gain). Generally, call four was focused on topics intended for previous weeks as participants regularly had less time to dedicate to each call than what was originally planned, making it difficult to cover the required number of topics. 


\section{Participant satisfaction}

Thirty-one percent $(n=44)$ participants completed the satisfaction survey. Respondents were very satisfied or satisfied overall $(93 \%, n=41)$, with the coaching quality $(98 \%, n=43)$ and the participant workbook (93\%, $n=41)$. Twenty-four participants (55\%) read all the participant workbook while $16(36 \%)$ read most of it.

Comments regarding improvements to the coaching and workbook were positive confirmations of the helpfulness of the program, dietitian counsellors and program flexibility. Participants suggested an electronic workbook (for ease of access when in a telecoaching call), smartphone app or additional videos could enhance the program.

Reported benefits of the program included feeling more positive about mindful eating and keeping on track with healthy eating and exercise. Many women felt they were better able to maintain their weight.

"I think I have managed to keep my weight within an acceptable amount and have definitely done more exercise than previous pregnancies"

Very few participants reported any dislikes or improvements for the program. A few participants would have liked additional meal plans while others wished that the program would continue past pregnancy.

"More resources on meal plan ideas (maybe there was I just didn't ask)"

\section{Effectiveness}

In the LWdP intervention group, $100 \%$ of women completed the baseline questionnaires. Follow-up behavioural questionnaires were complete for 45 women (32\%). There were no differences in age, country of birth, parity or total GWG for women who completed vs non-completers of questionnaires (results not shown). There were significantly more women who commenced pregnancy at an overweight BMI (47\% vs $29 \%$ ) and fewer women in the obese BMI category (36\% vs 54\%, P.046, results not shown) in the group who completed both questionnaires. Women who had completed the baseline and follow-up behavioural questionnaires attended more dietitian appointments ( $4.5 \pm 3.0$ vs $2.8 \pm 2.3, P=.003$, results not shown).

\section{Diet quality}

Total diet quality (Fat, Fibre and Total Index scores), and fruit and vegetable consumption improved significantly from baseline to follow-up (Table 2). The consumption of discretionary foods (take away, chocolate, chips etc) and sugar-sweetened beverages was reduced at follow up across all categories (Table 2). 
Table 2

FFBQ Index and dietary intake at baseline and follow up for women participating in the LWdP program

$\begin{array}{lll}\text { Baseline } & \begin{array}{l}\text { Follow- } \\ \text { up }\end{array} & \begin{array}{l}\text { Mean change }(95 \% \\ \mathrm{Cl}) \text { or }\end{array} \\ & & P \\ \% \text { change }\end{array}$

Fat and Fibre Behaviour Index

\begin{tabular}{llll} 
Total Index & $\begin{array}{l}3.03 \\
(0.45)\end{array}$ & $\begin{array}{l}3.53 \\
(0.36)\end{array}$ & 0.51 \\
Fat Index & $\begin{array}{l}3.01 \\
(0.59)\end{array}$ & $\begin{array}{l}3.51 \\
(0.47)\end{array}$ & 0.50 \\
& $\begin{array}{l}2.90 \\
(0.63)\end{array}$ & $\begin{array}{l}3.43 \\
(0.52)\end{array}$ & 0.50 \\
Fibre Index & & & \\
& & & \\
Serves of vegetables & 45 & 28 & \\
$1-4$ serves & $(100 \%)$ & $(62 \%)$ & \\
& $0(0 \%)$ & $\begin{array}{l}17 \\
(38 \%)\end{array}$ & $38 \%$ \\
\hline 5 or more serves* & & &
\end{tabular}

Serves of Fruit

0 - 1 serves

2 serves

3 serves

4 or more serves

Meets recommendations ( $2+$ serves)

Dairy

Usually/always choose low fat milk

Usually/ always choose low fat cream including ice-cream

Usually/ always chooses low fat cheese

Discretionary foods ( $\geq$ once/ week)

$15 \quad 5(11 \%)$

(33\%)

$19 \quad 25$

$(42 \%) \quad(56 \%)$

$11 \quad 15$

(24\%) (33\%)

$1(2 \%) \quad 0(0 \%)$

$31 \quad 40 \quad 20 \%$

$(69 \%) \quad(89 \%)$ $<.001$ 


\begin{tabular}{|c|c|c|c|c|}
\hline & Baseline & $\begin{array}{l}\text { Follow- } \\
\text { up }\end{array}$ & $\begin{array}{l}\text { Mean change }(95 \% \\
\mathrm{Cl}) \text { or } \\
\% \text { change }\end{array}$ & $P$ \\
\hline Takeaway & $\begin{array}{l}28 \\
(62 \%)\end{array}$ & $\begin{array}{l}10 \\
(22 \%)\end{array}$ & $-40 \%$ & $<.001$ \\
\hline Hot chips (French fries) & $\begin{array}{l}30 \\
(67 \%)\end{array}$ & $\begin{array}{l}13 \\
(29 \%)\end{array}$ & $-38 \%$ & $<.001$ \\
\hline Pastries, cakes, sweet biscuits & $\begin{array}{l}29 \\
(64 \%)\end{array}$ & $\begin{array}{l}19 \\
(42 \%)\end{array}$ & $-22 \%$ & .04 \\
\hline Chocolates or lollies & $\begin{array}{l}34 \\
(76 \%)\end{array}$ & $\begin{array}{l}23 \\
(51 \%)\end{array}$ & $-24 \%$ & .02 \\
\hline Sugar sweetened beverages & $\begin{array}{l}22 \\
(49 \%)\end{array}$ & $9(20 \%)$ & $-29 \%$ & $<.001$ \\
\hline Fruit juice or juice drinks & $\begin{array}{l}20 \\
(44 \%)\end{array}$ & $\begin{array}{l}12 \\
(27 \%)\end{array}$ & $-18 \%$ & .08 \\
\hline * missing numbers ( 15 baseline & & & & \\
\hline
\end{tabular}

\section{Intuitive eating scale}

Scores for the Intuitive Eating Scale-2 improved at follow-up on all measures except Unconditional permission to eat (Table 3).

Table 3

Intuitive Eating Scale scores with mean change at baseline and follow-up for women participating in the LWdP program

\begin{tabular}{|llll|}
\hline & Baseline & Follow-up & Mean change \\
\hline Unconditional permission to eat & $3.08(0.42)$ & $3.18(0.67)$ & $0.10(-0.13 .0 .32)^{*}$ \\
\hline Eating for physical rather than emotional reasons & $3.22(0.81)$ & $3.74(0.88)$ & $0.52(0.30,0.75)$ \\
\hline Reliance on internal hunger/ satiety cues & $3.21(0.77)$ & $3.53(0.85)$ & $0.32(0.12,0.52)$ \\
\hline Body-food choice congruence & $3.57(0.77)$ & $3.90(0.59)$ & $0.33(0.15,0.52)$ \\
\hline Total intuitive eating score & $2.39(0.94)$ & $3.53(0.64)$ & $0.14(-0.29,0.57)$ \\
\hline Differences between baseline and follow-up for all results $p<.001$ except for * $(p=.50)$ \\
\hline
\end{tabular}

\section{Physical activity and sedentary behaviour}


The minutes per week of all forms of physical activity increased from baseline to follow-up and the reported sedentary time spent on weekdays and weekends also decreased (Table 4). The number of participants meeting the physical activity guidelines increased at follow-up (62\% vs $75 \%, P=.17$ ).

Table 4

Physical activity and sedentary time before and after the LWdP program

\begin{tabular}{|lllc|}
\hline & Baseline & Follow-up & $P$ \\
\hline Total time (all PA, min/ week, median (IQR) & $180(90-250)$ & $240(140-310)$ & .007 \\
\hline Sedentary time weekdays, min/day (IQR) & $480(220-800)$ & $300(180-600)$ & .095 \\
\hline Sedentary time weekends, min/day (IQR) & $360(200-480)$ & $240(120-390)$ & .02 \\
\hline Physical activity guidelines & & & .37 \\
\hline Sufficient PA (>150min/week) & $28(62 \%)$ & $34(75 \%)$ & \\
\hline Insufficient PA (1- 149 min/week) & $13(29 \%)$ & $9(20 \%)$ & \\
\hline Sedentary (0 min/week) & $4(9 \%)$ & $2(4 \%)$ & \\
\hline
\end{tabular}

\section{Gestational weight gain}

Seventy-three percent $(n=30)$ of women in the historical comparison group and $70 \%(n=86)$ in the LWdP intervention group gained more weight than was recommended according to the IOM ( $P=.69$, Table 5). More women had met or exceeded the IOM weight recommendations prior to the first dietitian appointment in the historical comparison group $(n=23,47 \%)$ than the LWdP intervention group $(n=36$, $31 \%)(P=.10)$, though the number for both groups was high. The proportion of women exceeding the weight gain recommendations was similar between groups, regardless of ppBMI (Table 5). Between group comparison of women who attended at least 4 appointments (per protocol) gained average 12.2kg (6.3) vs $14.8 \mathrm{~kg}$ (7.5), $P=.47$ (Additional File 2). However, $10 \%(\mathrm{n}=5)$ of women achieved 'per protocol' in the historical comparison group, compared with $35 \%(n=50)$ in the LWdP intervention group (results not shown). 
Table 5

Gestational weight gain for women in the LWdP intervention group and historical comparison group.

\begin{tabular}{|llll|}
\hline & $\begin{array}{l}\text { LWdP intervention group } \\
(\mathbf{n = 1 4 2})\end{array}$ & $\begin{array}{l}\text { Historical comparison } \\
\text { group } \\
(\mathbf{n}=49)\end{array}$ & $\boldsymbol{P}$ \\
\hline Exceeded IOM guidelines for GWG* & $86(70 \%)$ & $30(73 \%)$ & .69 \\
\hline Normal weight & $16(67 \%)$ & $4(100 \%)$ & .33 \\
\hline Overweight & $31(72 \%)$ & $10(83 \%)$ & .43 \\
\hline Obese & $38(64 \%)$ & $16(64 \%)$ & .41 \\
\hline $\begin{array}{l}\text { Total gestational weight gain until } 36 \\
\text { weeks, kgs }\end{array}$ & $14.7(7.6)$ & $13.5(8.8)$ & .014 \\
\hline Normal weight & $18.6(4.6)$ & $26.9(10.3)$ & .64 \\
\hline Overweight & $15.9(6.7)$ & $17.1(9.5)$ & .07 \\
\hline Obese & $12.4(8.3)$ & $9.7(4.9)$ & \\
\hline *Adjusted for 36 weeks gestation & & & \\
\hline IOM institute of medicine; GWG gestational weight gain & & \\
\hline
\end{tabular}

Less women classified as obese before pregnancy, exceeded the IOM recommendations if they commenced the LWdP program before 16 weeks gestation (38\% vs $74 \%, P=.008$, Additional File 2 ). Referrer type (clinician vs self-referred) did not appear to influence the amount of GWG, except for women who commenced pregnancy in the normal BMl category where there was a trend towards a smaller proportion of women exceeding IOM GWG recommendations when they self-referred ( $50 \%$ vs $88 \%, P=.09$ ). Within the LWdP intervention group, the adjusted odds ratio of achieving weight gain according to the IOM guidelines was $0.883(95 \% \mathrm{Cl}, 0.386-2.02)$ (adjusted for parity, pre-pregnancy BMI, presence of oedema and weeks' gestation commencing LWdP $\leq 16$ weeks vs $>16$ weeks).

\section{Discussion}

This study has demonstrated the implementation of the LWdP program increased the referral of women eligible for pregnancy weight management support from one in thirty-three to one in one in eight, with demonstrated improvements in eating and activity behaviours in those who attended. However, despite a facilitated and deliberate implementation effort to integrate the service into antenatal care, the findings indicate further work is needed to ensure more women are provided with early intervention, particularly women with a high BMI at high risk of adverse pregnancy outcomes. 
Despite women not commencing the program until mid-way through their pregnancy, the implementation of $\mathrm{LWdP}$ resulted in a $10 \%$ increase in eligible women being referred for support during pregnancy and a three-fold increase in women receiving care. However, women's uptake of the program and retention of women was still poor. A similar evaluation of a telephone coaching lifestyle and weight management service during pregnancy, delivered external to the antenatal care health facility, observed less than one in ten women completing the program [33]. Based on previous research [34] the LWdP program was deliberately integrated into women's antenatal care where the dietitian health coach could see medical notes from other health care providers between appointments and document in medical records to allow other care providers to monitor progress. This alteration in referral and delivery approach resulted in a fourfold difference in completion compared with an external delivery strategy [33] with four in ten women completing the LWdP program.

While health professionals, predominantly midwives, were the primary source of referrals, a simple implementation strategy of a text message to all women registering for care at the facility resulted in a seven-fold increase in self referrals to the program. A consistent barrier reported by health professionals, particularly midwives is a lack of confidence and shame in discussing weight with women [35, 36], and the negative association women have with being referred to a dietitian [36,37]. This means many women who may benefit from intervention are never aware services exist to support them. Text message reminders have been commonly used to successfully increase attendance at health care appointments and adherence to behaviour change advice [38]. The LWdP program is one of the first to report the effectiveness of a text message prompt to encourage women to initiate their enrolment in a routine service, thus overcoming a key barrier to accessing support and increasing the reach of the program.

While the LWdP program delivered via telecoaching demonstrated equivalent GWG outcomes as achieved in the face-to-face care model of care, the proportion of women gaining more weight than recommended was high. This excess GWG may have been due to the relatively late recruitment of women to the program. Prior to commencing LWdP, one in three women had met or exceeded their recommended GWG for the entire pregnancy. First trimester GWG is recommended at around 1-2 kg regardless of ppBMI, with excess in early pregnancy being most closely associated with adverse outcomes including GDM [39], preeclampsia [40] and high birthweight [41]. Interestingly, women who commenced LWdP prior to 16-weeks gestation experienced lower GWG and a lower proportion of women who had a ppBMI in the obese range exceeded GWG recommendations. Furthermore, no additional benefits were noted towards achieving appropriate GWG for women attending per-protocol (4 or more appointments). These findings emphasise the importance of engaging women early in their pregnancy to support appropriate GWG, but to achieve this, barriers must be overcome. 'Late' entry to birthing facilities (usually 16 to 18 weeks) often results in a challenging situation of addressing this early excess GWG through lifestyle intervention when the rate of recommended GWG is greater [5]. Potential strategies to address these issues include aligning services with primary care settings to support early pregnancy lifestyle support [42] and providing tailored, individualised schedules of care to appropriately meet women's needs [43]. 
The improvements in dietary intake observed in women who participated in the LWdP program are consistent with previous interventions to support health behaviour change in pregnancy that impact on GWG $[44,45]$. Significant improvements in dietary quality were observed, driven by a reduction in discretionary food and an increase in fruit and vegetables. While there was no observed effect on GWG, high diet quality in pregnancy has been associated with a reduction in GDM, hypertension and pre-term birth [46]. Furthermore, if sustained, these improvements may contribute to a lowering of long-term diabetes and cardiovascular disease risk in women [47]. Somewhat unique to the LWdP was a focus on intuitive eating. The process of how women eat is likely to be as important, if not more so than, what women eat if long term behaviour change is sustained. Intuitive eating is considered an adaptive form of eating where there is a connection with internal hunger and satiety cues rather than emotions or cognitions driving food consumption [30,48]. Developing a healthy relationship with food where there is not a pre-occupation with dieting or the labelling of food as good or bad is needed before healthy eating can be pursued [48]. For many women experiencing a high body weight, breaking a long-held dieting cycle is likely to be important to sustaining behaviour change consistent with healthy eating and weight management. The improvements in intuitive eating observed within the program if sustained may assist women's eating behaviours well beyond the current pregnancy, having a long- term positive influence. This requires further investigation.

Positive changes in physical activity based on interventions during pregnancy have been mixed and vary according to the provision of supervised and structured activity [49]. The reduction in sedentary time and increase in overall duration of physical activity observed with the LWdP program based on counselling and behaviour change techniques, demonstrates that improved behaviour can be achieved independent of women needing to be provided with additional classes through birthing facilities.

There is limited guidance on the optimal duration, intensity, delivery method for the interventions to support behaviour change and healthy weight gain [10]. This may be because of the complex and individualised nature of lifestyle behaviour change and GWG. The LWdP program was specifically designed with continuity of health care to facilitate rapport and person-centred care, with call fidelity reducing as the call number progressed. Comments indicated women wanted to address topics relevant to them earlier than scheduled. Furthermore, a key factor in women withdrawing or not taking up LWdP was time and appointment burden. This is a common reason for not adopting weight management interventions in pregnancy, [33] and while remote delivery removes travel time, it does not address the need for another appointment. Collectively, the lack of evidence for what constitutes optimal care, and in practice the limited ability to meet women's needs by imposing a rigid schedule, point to the need for flexibility in services and modalities to deliver person centred care. Offering a suite of evidence-based options for remote delivery behaviour change support that can be individualised to each woman's circumstance may provide solutions engagement challenges, particularly to those women with a high body weight that may have more comorbidities requiring high risk pregnancy care.

\section{Strengths and limitations}


The findings of this study need to be considered in the context of several strengths and limitations. The completion of behavioural questionnaires by women was low and limited to those more likely to complete the program. The behavioural improvements observed are likely to reflect those women most motivated for change, it is possible that different approaches may be needed for women experiencing greater barriers to change or who are less motivated. Furthermore, the detailed program workbook and telephone delivery may have been a deterrent for women with a lower level of literacy. Future work needs to explore support options for those with lower education. The historical comparison group received face to face dietetic intervention, limiting the ability to determine the effectiveness of the LWdP program compared to no intervention. It is likely the differences observed in this evaluation would be more profound if compared to no intervention. However, in the context of overwhelming evidence and clinical guidelines recommending dietetic care for weight management support for women, this team deemed it unethical to withhold appropriate treatment. The dissemination methods of the staff survey meant we were unable to identify the total number approached to determine completion rate. A strength of this study was the strong theoretically driven approach, and the applied implementation within routine care demonstrated that a change in model of care is feasible within a large health service and results in favourable behaviour change for women who engage, with high participant satisfaction.

\section{Conclusions}

This is one of the few studies to report on the implementation of a telephone delivered behaviour change lifestyle program incorporated in routine antenatal care. It was developed to translate evidence from successful interventions into practice with facilitated implementation efforts specifically focussed on overcoming barriers to uptake and engagement. The intervention resulted in more women receiving care. Those women who engaged improved their eating and activity behaviours and intuitive eating and was no different than face to face care for GWG outcomes. The program requires adaptation to offer program enhancements such as technology that provide behaviour change support while reducing the appointment burden on women, while retaining the continuity of dietetic carer and the integration into antenatal care. For future scale up, a deeper understanding of how to engage those women with a prepregnancy BMI of $30 \mathrm{~kg} / \mathrm{m}^{2}$ and over earlier in pregnancy is needed to guide this implementation. Broader public health efforts are needed to focus efforts to reduce the prevalence of excess first trimester GWG for all women.

\section{List Of Abbreviations}




\begin{tabular}{|ll|}
\hline LWdP & Living Well during Pregnancy \\
\hline ppBMI & Pre-pregnancy body mass index \\
\hline GWG & Gestational weight gain \\
\hline IOM & Institute of Medicine \\
\hline GDM & Gestational diabetes mellitus \\
\hline FFBQ & Fat and Fibre Behavioural Questionnaire \\
\hline
\end{tabular}

\section{Declarations}

\section{Ethics approval and consent to participate}

All methods were carried out in accordance with relevant guidelines and regulations. Written informed consent was obtained from all participants in the LWdP program. Retrospective data collected for the historical control group was granted a waiver of consent under section 282 of the Public Health Act 2005 (RD007988). Ethics approval was granted from the Royal Brisbane and Women's Hospital Human Research Ethics Committee (HREC/17/QRBW/159).

\section{Consent for publication}

Not applicable

\section{Availability of data and materials}

The datasets analysed for the current study are available from the corresponding author on reasonable request and in accordance to local restrictions governing the privacy of information obtained from medical records.

\section{Competing interests}

The authors declare they have no competing interests.

\section{Funding}

$<$ blinded for peer review> is supported by a Metro North Health Clinician Research Fellowship. The Royal Brisbane and Women's Hospital Foundation Project Grant supported program evaluation.

\section{Authors' contributions}

SdJ, EE, SW, TG and TC were involved in the conception and design of the study. TG and HP contributed to data collection and reviewed the manuscript. NM performed the analysis. SdJ, NM, EE, LC, SW contributed to the interpretation of the works. SdJ and NM drafted the manuscript. EE, TC, ATC, SW, LC 
contributed to reviewing and revising the manuscript. All authors read, revised and approved the final manuscript.

\section{Acknowledgements}

The authors wish to acknowledge all women who participated in the evaluation of the Living Well during Pregnancy Program, clinical and administrative staff from Maternity Services at the Royal Brisbane and Women's Hospital.

\section{References}

1. Goldstein, R.F., et al., Association of gestational weight gain with maternal and infant outcomes: a systematic review and meta-analysis. Jama, 2017. 317(21): p. 2207-2225.

2. de Jersey, S.J., et al., A prospective study of pregnancy weight gain in A ustralian women. Australian and New Zealand Journal of Obstetrics and Gynaecology, 2012. 52(6): p. 545-551.

3. Kominiarek, M.A., et al., Association between gestational weight gain and perinatal outcomes. Obstetrics and gynecology, 2018. 132(4): p. 875.

4. Hedderson, M.M., E.P. Gunderson, and A. Ferrara, Gestational weight gain and risk of gestational diabetes mellitus. Obstetrics and gynecology, 2010. 115(3): p. 597.

5. Institute of Medicine and National Research Council, Weight Gain During Pregnancy: Reexamining the Guidelines, ed. a.A.L.Y. Kathleen M. Rasmussen. 2009: National Academies Press.

6. Gunderson, E.P., Childbearing and obesity in women: weight before, during, and after pregnancy. Obstetrics and Gynecology Clinics, 2009. 36(2): p. 317-332.

7. Gunderson, E.P. and B. Abrams, Epidemiology of gestational weight changes after pregnancy. Epidemiologic reviews, 2000. 22(2): p. 261-274.

8. Wrotniak, B.H., et al., Gestational Weight Gain and Risk of Overweight in the Offspring at Age $7 Y$ in a Multicenter, Multiethnic Cohort Study. The American journal of clinical nutrition, 2008. 87(6): p. 18181824.

9. International Weight Management in Pregnancy Collaborative Group, Effect of diet and physical activity based interventions in pregnancy on gestational weight gain and pregnancy outcomes: metaanalysis of individual participant data from randomised trials. BMJ (Clinical research ed.), 2017. 358: p. j3119-j3119.

10. Walker, R., et al., Attenuating pregnancy weight gain-what works and why: a systematic review and meta-analysis. Nutrients, 2018. 10(7): p. 944.

11. Bennett, C.J., et al., Interventions designed to reduce excessive gestational weight gain can reduce the incidence of gestational diabetes mellitus: A systematic review and meta-analysis of randomised controlled trials. Diabetes research and clinical practice, 2018. 141: p. 69-79.

12. Bailey, C., et al., Cost Effectiveness of Antenatal Lifestyle Interventions for Preventing Gestational Diabetes and Hypertensive Disease in Pregnancy. PharmacoEconomics-open, 2020. 4(3): p. 499-510. 
13. Harrison, C.L., et al., Weight management across preconception, pregnancy, and postpartum: $A$ systematic review and quality appraisal of international clinical practice guidelines. Obesity Reviews, 2021.

14. Health, Q., Queensland Clinical Guideline for the Management of Obesity in Pregnancy, Q. Health, Editor. 2015.

15. Lee, A., et al., Implementing a diet and exercise program for limiting maternal weight gain in obese pregnant women: A pilot study. Australian and New Zealand Journal of Obstetrics and Gynaecology, 2012. 52(5): p. 427-432.

16. Porteous, H., S. de Jersey, and M. Palmer, Attendance rates and characteristics of women with obesity referred to the dietitian for individual weight management advice during pregnancy. Australian and New Zealand Journal of Obstetrics and Gynaecology, 2020.

17. Byrne, C., et al., Poor attendance at group-based interventions for pregnancy weight management does location and timing matter? Nutrition \& Dietetics, 2017. 74(S1): p. 26.

18. Jimmieson, H., et al., Poor referral and completion of new dietary intervention targeting high maternal weight and excessive weight gain in pregnancy, does weight play a role? Nutrition \& Dietetics, 2017. 74(S1): p. 63.

19. Reeves MM, W.M., Brakenridge C, Goode AD, Winkler EAH, Fjeldsoe BS, Eakin EG. . Effectiveness of telephone-delivered interventions for achieving weight loss in overweight and obese adults: a metaanalysis. in 20th European Congress on Obesity. 2013. Liverpool.

20. Whelan, M.E., et al., Feasibility, effectiveness and cost-effectiveness of a telephone-based weight loss program delivered via a hospital outpatient setting. Translational Behavioral Medicine, 2015: p. 1-10.

21. Goode, A.D., M.M. Reeves, and E.G. Eakin, Telephone-Delivered Interventions for Physical Activity and Dietary Behavior Change.

22. Farzandipour, M., et al., Phone-based interventions to control gestational weight gain: a systematic review on features and effects. Informatics for Health and Social Care, 2018. 45(1): p. 15-30.

23. Harrison, C., et al., Preventing obesity across the preconception, pregnancy and postpartum cycle: Implementing research into practice. Midwifery, 2017. 52: p. 64.

24. Glasgow, R.E., T.M. Vogt, and S.M. Boles, Evaluating the public health impact of health promotion interventions: the RE-AIM framework. American journal of public health, 1999. 89(9): p. 1322-1327.

25. de Jersey, S., et al., Implementation of the Living Well During Pregnancy Telecoaching Program for Women at High Risk of Excessive Gestational Weight Gain: Protocol for an EffectivenessImplementation Hybrid Study. JMIR research protocols, 2021. 10(3): p. e27196.

26. Landes, S.J., S.A. McBain, and G.M. Curran, Reprint of: An introduction to effectivenessimplementation hybrid designs. Psychiatry research, 2020. 283: p. 112630.

27. Bandura, A., Health Promotion by Social Cognitive Means. Health Education and Behavior, 2004. 31(2): p. 143-164. 
28. Eakin, E.G., et al., Healthy Living after Cancer: a dissemination and implementation study evaluating a telephone-delivered healthy lifestyle program for cancer survivors. BMC cancer, 2015. 15(1): p. 992.

29. Reeves, M.M., E.A. Winkler, and E.G. Eakin, Fat and Fibre Behaviour Questionnaire: Reliability, relative validity and responsiveness to change in Australian adults with type 2 diabetes and/or hypertension. Nutrition \& Dietetics, 2015. 72(4): p. 368-376.

30. Tylka, T.L. and A.M. Kroon Van Diest, The Intuitive Eating Scale-2: item refinement and psychometric evaluation with college women and men. J Couns Psychol, 2013. 60(1): p. 137-53.

31. Australian Institute of Health and Welfare, The Active Australia Survey: A Guide and Manual for Implementation, Analysis and Reporting. 2004, AlHW: Canberra (AUST).

32. Craig, C.L., et al., International physical activity questionnaire: 12-country reliability and validity. Medicine \& science in sports \& exercise, 2003. 35(8): p. 1381-1395.

33. Rissel, C., et al., Piloting a telephone based health coaching program for pregnant women: a mixed methods study. Maternal and child health journal, 2019. 23(3): p. 307-315.

34. Seward, M.W., et al., Supporting healthful lifestyles during pregnancy: a health coach intervention pilot study. BMC pregnancy and childbirth, 2018. 18(1): p. 1-11.

35. Christenson, A., et al., Shame and avoidance as barriers in midwives' communication about body weight with pregnant women: A qualitative interview study. Midwifery, 2018. 63: p. 1-7.

36. Heslehurst, N., et al., Implementation of pregnancy weight management and obesity guidelines: a meta-synthesis of healthcare professionals' barriers and facilitators using the $T$ heoretical $D$ omains F ramework. Obesity Reviews, 2014. 15(6): p. 462-486.

37. Atkinson, L., E.K. Olander, and D.P. French, Why don't many obese pregnant and post-natal women engage with a weight management service? Journal of Reproductive and Infant Psychology, 2013. 31(3): p. 245-256.

38. Schwebel, F.J. and M.E. Larimer, Using text message reminders in health care services: a narrative literature review. Internet interventions, 2018. 13: p. 82-104.

39. MacDonald, S.C., et al., Patterns of gestational weight gain in early pregnancy and risk of gestational diabetes mellitus. Epidemiology (Cambridge, Mass.), 2017. 28(3): p. 419.

40. Bodnar, L.M., et al., Early-pregnancy weight gain and the risk of preeclampsia: A case-cohort study. Pregnancy hypertension, 2018. 14: p. 205-212.

41. Davenport, M.H., et al., Timing of excessive pregnancy-related weight gain and offspring adiposity at birth. Obstetrics \& Gynecology, 2013. 122(2 PART 1): p. 255-261.

42. Walker, R., et al., Barriers and enablers of implementing gestational weight gain guidelines in GP-led shared maternity care: a qualitative study. Proceedings of the Nutrition Society, 2018. 77(OCE4).

43. Susan, J., et al., Prospective relationships between health cognitions and excess gestational weight gain in a cohort of healthy and overweight pregnant women. Journal of the Academy of Nutrition and Dietetics, 2017. 117(8): p. 1198-1209. 
44. Flynn, A.C., et al., Dietary patterns in obese pregnant women; influence of a behavioral intervention of diet and physical activity in the UPBEAT randomized controlled trial. International Journal of Behavioral Nutrition and Physical Activity, 2016. 13(1): p. 1-12.

45. Dodd, J.M., et al., The effects of antenatal dietary and lifestyle advice for women who are overweight or obese on maternal diet and physical activity: the LIMIT randomised trial. BMC medicine, 2014. 12(1): p. 1-19.

46. Yu, Y., et al., Association between diet quality during preconception or pregnancy and adverse perinatal outcomes: a systematic review and meta-analysis. Authorea Preprints, 2021.

47. McNaughton, S.A., et al., Dietary quality is associated with diabetes and cardio-metabolic risk factors. The Journal of nutrition, 2009. 139(4): p. 734-742.

48. Van Dyke, N. and E.J. Drinkwater, Review article relationships between intuitive eating and health indicators: literature review. Public health nutrition, 2014. 17(8): p. 1757-1766.

49. Flannery, C., et al., Effectiveness of physical activity interventions for overweight and obesity during pregnancy: a systematic review of the content of behaviour change interventions. International Journal of Behavioral Nutrition and Physical Activity, 2019. 16(1): p. 1-20.

\section{Figures}




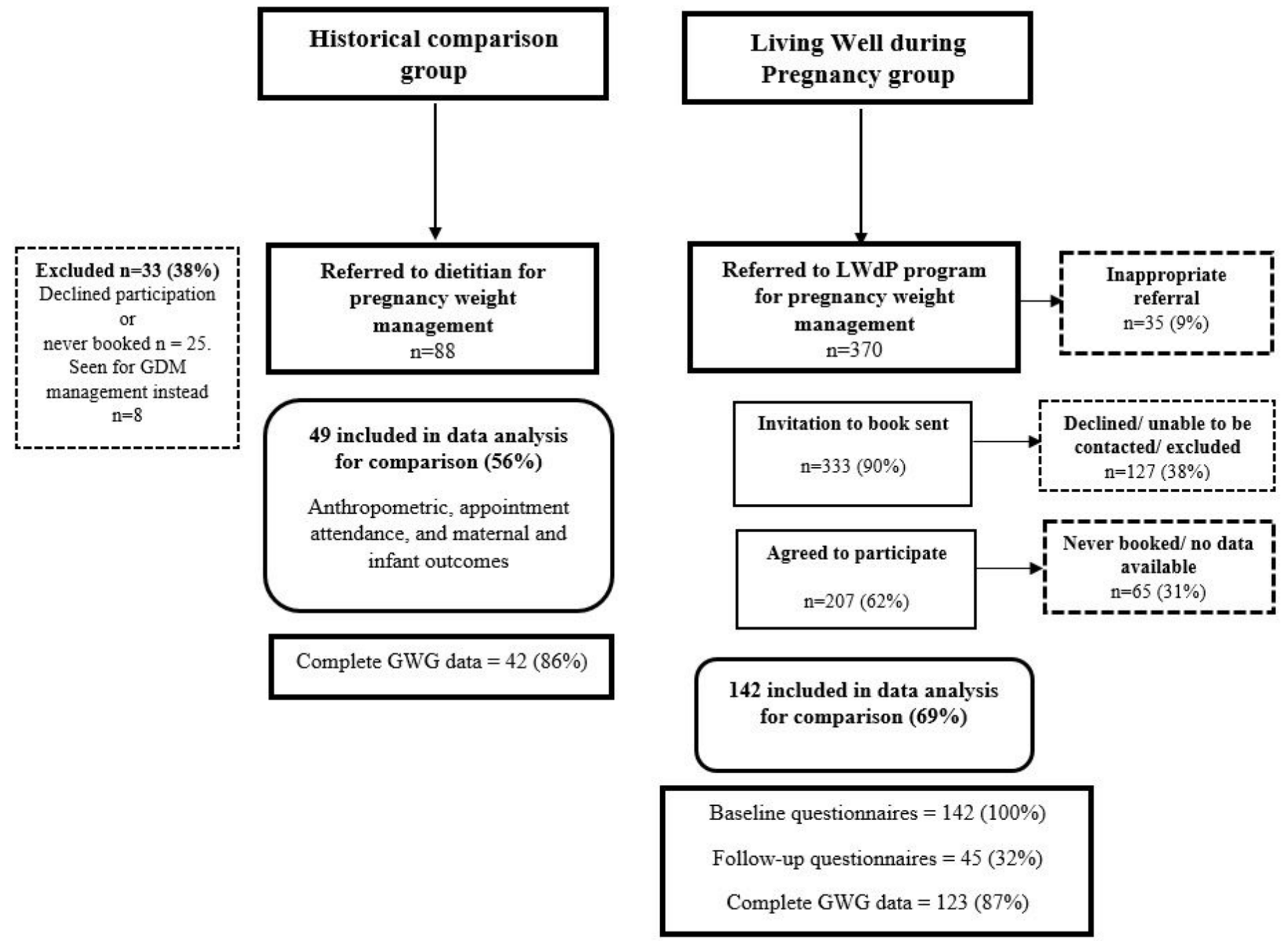

Figure 1

Study flow diagram (reach) of historical comparison and LWdP groups. Percentages relate to the number of participants in the previous level of the flow chart

\section{Supplementary Files}

This is a list of supplementary files associated with this preprint. Click to download.

- AdditionalFile1ImplementationStrategies.docx

- AdditionalFile2AdditionalGWGdata.docx 